\section{La fractura vertebral: una entidad en busca de definición}

\author{
Manuel Sosa Henríquez \\ Unidad Metabólica Ósea. Hospital Universitario Insular. \\ Universidad de Las Palmas de Gran Canaria.
}

\section{Fractura vertebral}

Por su importante prevalencia, a la osteoporosis se le ha denominado la epidemia silenciosa del siglo $\mathrm{XXI}^{1}$, pues se estima que, a partir de los 50 años, una mujer de raza blanca tiene aproximadamente un $40 \%$ de posibilidades de sufrir alguna fractura en el resto de su vida, riesgo que también comparte un varón de la misma edad, aunque es sensiblemente menor, en concreto del $13 \%^{2}$. La magnitud de esta epidemia es también considerable si se define la osteoporosis por criterios densitométricos, cuando aún no se ha producido la fractura, lo cual por otra parte sería lo ideal. Así, en la población española se estima que el $40 \%$ de las mujeres de más de 70 años y el 11,3\% de los varones de la misma edad presentan esta enfermedad ${ }^{3,4}$.

Volviendo a las fracturas osteoporóticas, la fractura vertebral (FV) posee unas connotaciones especiales. Se ha publicado que es la fractura más frecuente y esto lo corroboró el estudio EVOS (European Vertebral Osteoporosis Study), un trabajo cooperativo multicéntrico europe $0^{5}$ donde se constató que en la población europea de 50 o más años existía al menos una FV en el $20,2 \%$ de las mujeres y en el $12 \%$ de los varones. En España, los resultados no fueron muy diferentes del resto de Europa, observándose en nuestra población femenina al menos una FV entre el 14,9\% de Madrid y el $26 \%$ de Barcelona, y en los varones entre el $19,8 \%$ de Madrid y el $25,1 \%$ de Las Palmas ${ }^{6}$. Además, desde hace tiempo parece existir acuerdo para aceptar que la FV es una causa importante de dolor ${ }^{7}$, de deformidad ${ }^{8}$ e incluso de deterioro de la calidad de vida ${ }^{9}$. En algunos estudios se ha establecido que la existencia de una FV previa constituye en sí misma un riesgo para la aparición de nuevas FV ${ }^{10}$.

Las cifras de prevalencia de la FV en Europa, en el momento de su publicación, resultaron sorprendentes en el varón, ya que previamente Mann et al ${ }^{11}$ al habían obtenido en una población similar de americanos una prevalencia de FV del $10 \%$. Inmediatamente surgen algunas preguntas. ¿Las diferencias son reales y, si es así, a qué se deben? 0 bien, ¿existe un problema de metodología y no se está midiendo lo mismo?

Y aquí es donde reside el problema. En el diagnóstico de la FV. En ésta no existe una línea de fractura, por lo que para su calificación como tal es necesaria la utilización de unos criterios morfométricos, basados en la mayor parte de las ocasiones en las mediciones de la longitud del cuerpo vertebral en su porción anterior, media y posterior, y compararlas con las obtenidas en las vértebras adyacentes por medio de diferentes fórmulas, cocientes o porcentajes o con valores de normalidad previamente establecidos. Existen varios mé-

Correspondencia: Dr. M. Sosa Henríquez. Unidad Metabólica Ósea.

Departamento de Ciencias Médicas y Quirúrgicas.

Universidad de Las Palmas de Gran Canaria.

Apartado 550. 35080 Las Palmas de Gran Canaria.

Correo electrónico: msosa@cicei.ulpgc.es

Recibido el 20-9-2000; aceptado para su publicación el 10-10-2000

Med Clin (Barc) 2000; 115: 661-662 todos cualitativos o cuantitativos. Combinando todo esto, finalmente, cabe citar que se han publicado al menos una docena de criterios para definir la $\mathrm{FV}^{12}$, lo que indica que ninguno es concluyente. Por otra parte, los términos «deformidad» y «fractura vertebral» en ocasiones se superponen y utilizan de forma indistinta, lo que contribuye aún más a la confusión. Sin embargo, desde nuestro punto de vista, el problema más importante lo constituye la variabilidad tan amplia en los resultados según se aplique un criterio u otro. Así, en la población canaria que participó en el estudio EVOS, aplicando el método de Eastell se obtenía una prevalencia de FV del $25,1 \%$ en los varones y del $22,7 \%$ en las mujeres, mientras que aplicando el criterio de McCloskey la prevalencia era del $10,8 \%$ en el caso de las mujeres, menos de la mitad 6 .

Esto ha motivado que últimamente se esté asistiendo a una especie de "revolución» en lo que respecta a lo que es verdaderamente la FV, habiéndose publicado algunos artículos que Ilegan a cuestionar muy seriamente no sólo su identidad, sino también su importancia clínica. En una reciente revisión, no se insinúa, sino que se dice claramente y sin medias tintas que las FV pueden estar utilizándose de manera fraudulenta, que han sido fabricadas por investigadores listos para: a) facilitar el reclutamiento de pacientes en los estudios diseñados a fin de probar nuevos fármacos en el tratamiento de la osteoporosis, y b) como un criterio de eficacia en estos estudios, menos riguroso que las fracturas clínicas $^{13}$. Como apoyo de esta teoría se señala que el $65 \%$ de los pacientes con estas moderadas deformidades están asintomáticos ${ }^{14}$, que existe una escasa correlación entre la densidad mineral ósea y estas deformidades vertebrales ${ }^{15}$, y que la presencia de estas deformidades se asocia a valores densitométricos que están tan sólo un $5 \%$ por debajo de los valores medios de los controles, por lo que no deberían ser tratadas como fracturas reales sino como factores de riesgo. Concluye este autor, desde el anonimato, que es necesario que existan al menos dos o tres deformidades vertebrales para que la densidad mineral ósea se encuentre en estas mujeres en el rango de osteoporosis y tres deformidades en el caso de los varones ${ }^{13}$.

En medio de toda esta confusión, en este mismo número de Medicina CLínicA, Naves et al ${ }^{16}$ aportan algo de luz, publicando los resultados del seguimiento de la población que participó en el estudio EVOS en Asturias, durante un período de 6 años, confirmando, por una parte, que la FV es la fractura osteoporótica que tiene una mayor incidencia y que su presencia constituye un importante factor de riesgo en la aparición de nuevas fracturas tanto vertebrales como de cadera. Estos resultados coinciden plenamente con los descrito por Black et $\mathrm{al}^{17}$. El trabajo es metodológicamente impecable y se trata del primer estudio español con un seguimiento tan largo, mientras que sus limitaciones, escasas, son comentadas por los mismos autores en la discusión. Quizá la principal limitación sea que los datos sobre la incidencia de nuevas $\mathrm{FV}$ a los 6 años se obtienen a partir de algo más de la mitad de la población que inició el estudio, concretamente el $55 \%$; pero esto es inevitable en este tipo de estudios y puede deberse al hecho de que la población se termina cansando o bien preocupando por estudios epidemiológicos («no indicados por su médico, sino para investigar») en los que reciben rayos $X$ aunque sea en pequeñas dosis.

No obstante, y ahondando algo más en lo ya comentado, Naves et $a^{16}$ utilizan para definir la FV el criterio de Genant, cuando precisamente en esta misma población, en el estudio EVOS, se utilizaron otros criterios, concretamente los de Eastell y McCloskey. Esto no invalida en absoluto el trabajo, pero pone de manifiesto, una vez más, la existencia de dife- 
rentes opiniones y procedimientos y, sin duda, establece la necesidad de obtener una uniformidad en el diagnóstico de lo que es una FV. Con este fin, en el único documento de consenso existente hasta el momento por autores españoles dedicados monográficamente al campo de las enfermedades metabólicas óseas y auspiciado tanto por la Sociedad Española de Osteoporosis y Metabolismo Mineral (SEIOMM) como por la Fundación Hispana para la Osteoporosis y Enfermedades Metabólicas Óseas (FHOEMO) ${ }^{18}$, se aconseja precisamente la utilización del índice de deformidad vertebral de Genant.

Actualmente, los adelantos científicos se suceden a una velocidad vertiginosa y esto quizá no permite sedimentar nuestros conocimientos y aplicarlos con mayor serenidad y reflexión. Cuando aún no terminamos de conocer bien una determinada técnica aparece otra nueva a la que hay que prestar atención, pues de no hacerlo se correría el riesgo de quedarnos rezagados, pero sin saber con certeza si es mejor que la anterior. Y esto ocurre también en el campo de las enfermedades metabólicas óseas ${ }^{19}$. Disponemos de un gran número de métodos para determinar la masa ósea, marcadores bioquímicos de remodelamiento óseo, fármacos para el tratamiento de la osteoporosis y, cómo no, criterios de fractura vertebral.

\section{REFERENCIAS BIBLIOGRÁFICAS}

1. Fogelman I, Ryan P. Osteoporosis: a growing epidemic. Br J Clin Pract 1991; 45: 189-196.

2. Melton III LJ, Atkinson EJ, O'Fallon WM, Wahner HW, Riggs BL. Longterm fracture risk prediction with bone mineral measurements made at various skeletal sites J Bone Miner Res 1991; 6 (Supl 1): 136.

3. Díaz Curiel M. Prevalencia de la osteoporosis densitométrica en la población española. En: Díaz Curiel M, Díez Pérez A, Gómez Alonso C, editores. Nuevas fronteras en el estudio de la densidad ósea en la población española. Madrid: Rhone Poulenc Rorer, 1996.

4. Díaz Curiel M, Turbí Disla C, Rapado A, García JJ. Prevalencia de osteopenia y osteoporosis densitométrica en la población masculina española. Rev Esp Enf Metab Oseas 1997; 6: 129-132.
5. Silman AJ, y el EVOS Study Group. The European Vertebral Osteoporosis Study Group. (EVOS). En: Christiansen C, Riis B, editores. Osteoporosis. Proceedings of The Fourth International Symposium on Osteoporosis and Consensus Development Conference, Rødovre, 1993; 65-66.

6. O'Neill TW, Felsenberg D, Varlow J, Cooper C, Kanis JA, Silman AJ, y el European Vertebral Osteoporosis Study Group (EVOS). The prevalence of vertebral deformity in European men and women: the European Vertebral Osteoporosis Study. J Bone Miner Res 1996; 11: 1010-1018.

7. Ismail AA, Cooper C, Felsenberg D, Varlow J, Kanis JA, Silman AJ et al. Number and type of vertebral deformities: epidemiological characteris tics and relation to back pain and height loss. Osteoporosis Int 1999; 9 : 206-213.

8. Lau EMC, Woo J, Chan H, Griffith J, Chan YH, Leung PC. The health consequences of vertebral deformity in elderly Chinese men and women. Calcif Tissue Int 1998; 63: 1-4.

9. Pluijm SMF, Tromp AM, Smit JH, Deg DJH, Lips PJ. Consequences of vertebral deformities in older men and women. J Bone Miner Res 2000; 15: 1564-1572

10. Melton LJ III, Atkinson EJ, Cooper C, O'Fallon WM, Riggs BL. Vertebral fractures predict subsequent fractures. Osteoporosis Int 1999; 10: 214221.

11. Mann T, Ovita SK, Wilson D, Nelson D, Orwoll ES. Vertebral deformity in men. J Bone Miner Res 1992; 7: 1259-1265.

12. Nelson DA, Kleerekoper M. What is a vertebral fracture? En: Marcus R, Feldman D, Kelsey J, editores. Osteoporosis. San Diego: Academic Press, 1996; 613-621.

13. Anónimo. Vertebral deformation: fake fractures fuel folly. Lunar News 2000; 1: 4-6.

14. Melton III LJ, Lane AW, Cooper C, Eastell R, O'Fallon WM, Riggs BL. Prevalence and incidence of vertebral deformities. Osteoporosis Int 1993; 3: 113-119.

15. Nevitt MC, Ross PD, Palermo L, Musliner T, Genant HK, Thompson DE. Association of prevalent vertebral fractures, bone density and alendronate treatment with incident vertebral fractures: effect of number and spinal location of fractures. Bone 1999; 25: 613-619.

16. Naves Díaz ML, Díaz López B, Gómez Alonso C, Altadill Arregui A, Rodríguez Rebollar A, Cannata Andía JB. Estudio de incidencia de fracturas osteoporóticas en una cohorte mayor de 50 años durante un período de 6 años de seguimiento. Med Clin (Barc) 2000; 115: 650-653.

17. Black DM, Arden NK, Palermo L, Pearson J, Cummings SR. Prevalent vertebral deformities predicts hip fractures and new vertebral deformities but not wrist fractures. J Bone Miner Res 1999; 14: 821-828.

18. Sosa Henríquez M, editor. El Documento Canario sobre la Osteoporosis. Barcelona: Ediciones Permanyer, 1998.

19. Sosa Henríquez M. Estimado Dr. Albright. Rev Esp Enf Metab Oseas 2000; 9: 133-134 